\title{
Exploring Genetics Influences on Birth Weight
}

\author{
Sam Trejo ${ }^{1^{*}}$
}

1. University of Wisconsin-Madison, School of Public Affairs \& Department of Sociology

*Send correspondence to sam.trejo@wisc.edu.

August 2020

\section{Acknowledgements}

This work has been supported by the National Science Foundation (DGE-1656518), the Institute of Education Sciences (R305B140009), the Russell Sage Foundation (96-17-04), and National Institute on Aging (P30AG017253). We are grateful to Benjamin Domingue, Liam Hill, Dalton Conley, Jeremy Freese, Tom Dee, David Rehkopf, and Brian Jacob for helpful comments. We also wish to thank Dan Mason, Rosie McEachan, and the Born and Bradford (BiB) staff as well as Pamela Herd and the Wisconsin Longitudinal Study (WLS) staff for access to the restricteduse genetic data. BiB receives core infrastructure funding from the Wellcome Trust (WT101597MA) and the National Institute for Health Research (NIHR) under its Collaboration for Applied Health Research and Care (CLAHRC) for Yorkshire and Humber and Clinical Research Network (CRN) research delivery support. Further support for genome-wide and multiple 'omics measurements is from the UK Medical Research Council (G0600705), National Institute of Health Research (NF-SI-0611-10196), US National Institute of Health (R01 DK10324), and the European Research Council under the European Union's Seventh Framework Programme (FP7/2007-2013) / ERC grant agreement no 66954. The WLS is directed by Pamela Herd, and the work conducted herein was supported by the National Institute on Aging (R01 AG041868-01A1 and P30 AG017266). This research benefitted from GWAS results made available by the Early Growth Genomics Consortium. All funders had no role in the design of the study, the collection, analysis, or interpretation of the data; the writing of the manuscript, or the decision to submit the manuscript for publication. Any opinions expressed are those of the authors alone. 


\begin{abstract}
A person's birth weight is a robust predictor of their economic, educational, and health outcomes across the life course. But is birth weight simply a proxy for environmental conditions in-utero, or do biological processes related to birth weight themselves play an important role in healthy development during the prenatal period? To examine this question, we utilize an underexplored source variation in birth weight that is largely orthogonal to prenatal environmental conditions: an individual's genes. We leverage the recent identification of specific genetic variants associated with birth weight to construct polygenic scores in two longitudinal studies and empirically validate and unpack the molecular genetic correlates of birth weight. A 1 SD increase in the birth weight polygenic score is associated with a $\sim 100$ gram increase in birth weight; sibling comparisons show this association mostly represents the causal effect of genes. The magnitude of the relationship between polygenic score and birth weight is increased for children who spend longer in the womb and children born to mothers with high maternal body mass index. Finally, the birth weight polygenic score causally affects downstream social and cognitive outcomes, providing support for the hypothesis that birth weight itself is intimately related to healthy prenatal development.
\end{abstract}

\title{
Keywords
}

Fetal Origins, Birth Weight, Polygenic Score, Life Course Development, Biodemography 


\section{Introduction}

Over the last two decades, the social and biomedical sciences have witnessed an explosion of research on the fetal origins hypothesis, also referred to as the developmental origins of health and disease (Almond, Currie, and Duque 2018; Almond and Currie 2011; Victora et al. 2008; Barker 1990; 1995). A large body of work has demonstrated that an individual's birth weight is robustly associated with their economic, educational, and health outcomes later in life. ${ }^{1}$ This research affirms the importance of healthy development during the prenatal period. However, is birth weight itself important per se, or does birth weight simply act as a coarse proxy for prenatal environmental conditions (Conti et al. 2020)? To examine this question, we leverage an underexplored source variation in birth weight — one is that is largely orthogonal to the prenatal environment: an individual's genes. ${ }^{2}$

In Figure 1, we present two plausible stylized causal models linking genes, the prenatal environment, birth weight, and downstream development. In Panel A, birth weight simply acts as an index of prenatal environmental exposures. Aspects of the prenatal environment, such as exposure to malnutrition and stress, simultaneously affect both birth weight and valued developmental outcomes. In this case, because birth weight and development are related only through the prenatal environment, genes that influence birth weight do not affect outcomes later

\footnotetext{
${ }^{1}$ See also Almond, Chay, \& Lee 2005; Behrman \& Rosenzweig 2004; Black, Devereux, \& Salvanes 2007; Boardman, Powers, Padilla, \& Hummer 2002; Conley \& Bennett 2000; Conley, Strully, \& Bennett 2006; Figlio, Guryan, Karbownik, \& Roth 2014; Oreopoulos, Stabile, Walld, \& Roos 2008; Royer 2009; Torche \& Echevarría 2011.

${ }^{2}$ Among unrelated individuals, genetic influences on birth weight are orthogonal to environmental influences on birth weight only in the sense that they represent a distinct causal pathway (though a non-zero correlation may still exist between an individual's genes for birth weight and aspects of their prenatal environment nonetheless). However, among siblings, genetic variation related to birth weight is randomly assigned and therefor mechanically orthogonal to prenatal environmental conditions.
} 
in life. In Panel B, on the other hand, birth weight is itself an important part of the prenatal development process. That is, birth weight mediates the relationship between prenatal environmental exposures and developmental outcomes. Here, genes that influence birth weight in turn impact development across the life course.

\section{[Insert Figure 1 Here]}

An increased understanding of the role that genetic influences on birth weight play in shaping developmental outcomes would help adjudicate between the two potential causal models for prenatal human capital formation presented in Figure 1. However, to date, genetic influences on birth weight are largely absent from the fetal origins literature. Genes are typically either relegated to the error term or differenced out entirely using a quasi-experimental identification strategy (Wooldridge 2015; Papageorge and Thom 2020). ${ }^{3}$ Thus, precious little is known about the ways in which genes influence birth weight and whether genetic influences on birth weight translate into positive outcomes later in life.

Fortunately, advances in the field of molecular genetics have recently introduced novel avenues for explicitly modeling genetic influences on human health and behavior (Beauchamp et al. 2011; Benjamin et al. 2012; Harden and Koellinger 2020; Mills and Tropf 2020). Last year, a large study combined the DNA of hundreds of thousands of participants from across the globe and successfully identified specific genetic variants associated with birth weight (Warrington et al. 2019). In this paper, we encapsulate these identified genetic variants related to birth weight into a single individual-level linear predictor known as a polygenic score (Dudbridge 2013). We

${ }^{3}$ In the case of birth weight, comparing monozygotic twins with a family fixed effects model has become the predominant approach. 
then use this birth weight polygenic score in two longitudinal studies to i) validate molecular genetic discoveries related to birth weight and ii) develop an improved understanding of the causal model that connects the prenatal period to birth weight to outcomes across the life course.

We examine three specific questions regarding genetic influences on birth weight. First, we ask: (1) Do individual genetic factors—summarized by a new polygenic score — predict birth weight and does this association in part represent a causal effect? To do so, we test whether the polygenic score is associated with birth weight a) between families using both generic linear models fit on unrelated individuals and b) within families using family fixed effects models fit on sibling pairs. Because genetic differences between siblings are randomly assigned (Conley and Fletcher 2017), comparing differences in polygenic scores between siblings to differences in outcomes between siblings (Rietveld et al. 2014; Domingue et al. 2015; Lee et al. 2018; Belsky et al. 2018; Trejo and Domingue 2019; Brumpton et al. 2020; Zaidi and Mathieson 2020) allows us to test whether the genetic correlates of birth weight are causally related to outcomes. Next, we test whether genetic sources of variation in birth weight, like environmental sources in variation, are related to downstream social and cognitive development. We ask: (2) Do genetic factors related to birth weight predict social and cognitive development across the life course, and what does this tell us about the nature of the relationship between birth weight and healthy development? Finally, we probe for gene-environment interplay-whether, genetic and environmental influences may be interactive, rather than additive, in how they shape an individual's birth weight (Armstrong-Carter et al. 2019; Barcellos, Carvalho, and Turley 2018; Papageorge and Thom 2020; Boardman et al. 2014). We ask: (3) Do environmental factors moderate the relationship between a child's genes and their birth weight? 
We find that a one standard deviation increase in an child's birth weight polygenic score is associated with a $~ 100$ gram increase in their birth weight; within family analyses leveraging genetic differences between siblings show that a large portion of this association represents the causal effect of genes. The birth weight polygenic score also predicts social and cognitive outcomes starting in childhood through adulthood, both within and between families. Finally, the relationship between polygenic score and birth weight is increased for children who spend longer in the womb and children born to mothers with high maternal body mass index. Our findings support the idea that recent efforts have identified a relatively accurate—albeit noisy—map linking an individual's DNA sequence to their genetic predisposition to weigh more at birth, but that nonetheless an individual's genetic predisposition varies across contexts. Moreover, our results are inconsistent with the idea that birth weight simply acts as a proxy for prenatal environmental conditions. Instead, they suggest that the biological processes related to birth weight themselves play an important role in healthy prenatal development.

\section{Molecular Genetics and Complex Traits}

In this section, we review the relevant literature on recent molecular genetic discoveries. Previously, scientists were greatly limited in their capacity to engage with individual genetic differences. Even upon the completion of the Human Genome Project (Lander et al. 2001), only small quantities of genetic information were available for scientific inquiry. Over the last 10 years, however, growing sample sizes of genotyped individuals and computational developments in the processing of large amounts of genetic data have led to substantial developments in detecting replicable associations between genes and various social outcomes. 


\section{2a. Genome-Wide Association Studies}

The advent and proliferation of genome-wide association studies (GWAS) has ushered in new understanding of the genetics of complex traits and the biology of human behavior and disease (Visscher et al. 2012; 2017). GWAS probe the relationship between traits and regions of the genome via large data sets containing individual-level information on genotype and phenotype (Pearson and Manolio 2008). GWAS rely on the availability of DNA from hundreds

of thousands of individuals and identify variants of single-nucleotide polymorphisms (SNPs) that are more likely to be found among people exhibiting a trait of interest. SNPs are the most common form of human genetic variation and represent a mutation at a single locus in the genome; GWAS typically involve analyzing millions of SNPs.

Though GWAS methodologies vary slightly, in general they involve regressing some outcome $Y_{i}$ on a person's allele frequency $g_{i}^{n}$ iteratively for $N$ independent SNPs. GWAS also typically control for sex, age, and the first 10 principal components (PCs) of individual genotype.

$$
Y_{i}=\hat{\delta}^{n}+\hat{\alpha}^{n} g_{i}^{n}+\boldsymbol{X}_{\boldsymbol{i}} \widehat{\boldsymbol{\Theta}}+\epsilon_{i}
$$

$Y_{i}$ : GWAS outcome for individual $i$

$\hat{\alpha}^{n}$ : Observed linear association between a one allele change at a individual's genetic locus $n$ and outcome $Y$ $g_{i}^{n}$ : Total number of risk alleles at individual $i^{\prime}$ s genetic locus $n(0,1$, or 2$)$

$\boldsymbol{X}_{\boldsymbol{i}}$ : Vector of individual $i$ covariates including sex, age, and the first 10 PCs of genotype

To date, GWAS have been conducted on a wide variety of outcomes, ranging from proximal, biological phenotypes, such as blood pressure (Giri et al. 2019) and height (Yengo et 
al. 2018), to distal, behavioral phenotypes, such as depression (Hyde et al. 2016; Okbay et al. 2016), educational attainment (Lee et al. 2018), and risk tolerance (Linnér et al., 2019).

\section{2b. Theoretical and Observed Polygenic Scores}

A key discovery from the first decade of GWAS is that the vast majority of characteristics studied by social and behavioral scientists are complex traits (Visscher et al. 2012; 2017). A complex trait is one influenced by a large number of genes, with each gene having only a tiny effect (Chabris et al. 2015; Boyle, Li, and Pritchard 2017). Thus, the GWAS literature has shown that the genetic influence on most behavioral traits is due to many genes rather than a small number of genes or a single gene.

To increase statistical power and simplify computation, researchers often generate an individual-level linear predictor called a polygenic score (Dudbridge 2013). A polygenic score is meant to summarize all the identified genetic information dispersed widely across the genome that pertains to a particular trait. Such scores have become widely utilized (Duncan et al. 2019) and rely on the assumption that SNP-level genetic effects are linear and additive. ${ }^{4}$

The theoretical (though unobserved) polygenic score of person $i$ can be expressed mathematically as the summation of the product between their allele count and the true SNPlevel causal effect, or allelic weight, across $N$ independent genetic loci.

$$
\sum_{n=1}^{N} \alpha^{n} g_{i}^{n}=P G S_{i}
$$

(2b.i)

\footnotetext{
${ }^{4}$ Recent meta-analyses of twin studies support the linear, additive model for genetic effects (Polderman et al. 2015).
} 
$\alpha^{n}$ : True causal effect of a one allele change at a individual's genetic locus $n$ on outcome $Y$ $P G S_{i}$ : Polygenic score constructed from the true causal linear effect of a individual's genes on outcome $Y$

In practice, the vector of true causal effects at all $N$ independent genetic loci, $\vec{\alpha}$, is unobserved. Instead, we obtain $\overrightarrow{\hat{\alpha}}$, an estimate of $\vec{\alpha}$, by fitting a GWAS (see Equation 2a.i). We can then use $\overrightarrow{\hat{\alpha}}$ to construct our observed polygenic score, $\widehat{P G} S_{i}$, in an independent sample.

$$
\sum_{n=1}^{N} \hat{\alpha}^{n} g_{i}^{n}=\widehat{P G} S_{i}
$$

$\widehat{P G} S_{\mathrm{i}}$ : Polygenic score constructed from the observed linear relationship between a person's genes and outcome $Y$

$\widehat{P G} S_{i}$ is then normalized within sample to have mean 0 and standard deviation 1 . The observed polygenic score, $\widehat{P G} S_{i}$, can be thought of as a noisy version of the true polygenic score $P G S_{i}$

$$
\widehat{P G S} S_{i}=\sum_{n=1}^{N}\left(\hat{\alpha}^{n}+\mu^{n}\right) g_{i}^{n}=P G S_{i}+\mu_{i}
$$

$\mu^{n}$ : Difference between true and observed allelic weight at loci genetic locus $n$ due to finite GWAS sample $\mu_{i}$ : Measurement error of undefined form

At present, the $\mu_{i}$ component of observed polygenic scores for most social and behavioral traits tends to be quite large, causing substantial attenuation bias towards zero. For example, 
previous studies suggest that $P G S_{i}$, the true, unobserved polygenic score for birth weight, would explain $\sim 40 \%$ of the variance in birth weight among unrelated individuals (Warrington et al. 2019). However, due to the finite sample used to estimate $\overrightarrow{\hat{\alpha}}$, the observed polygenic score used in this study explains only 3\% of the variance in birth weight (Authors' Calculations, Born in Bradford cohort).

\section{2c. GWAS and Birth Weight}

Birth weight, like virtually all human characteristics (Polderman et al. 2015), is determined by a combination of genetic and environmental factors. A recent study conducted GWAS on the maternal and fetal genetic influences on birth weight using data on over 500,000 individuals and revealed new insights into the genetic underpinnings of birth weight (Warrington et al. 2019). The authors identified 190 independent regions of the genome that are significantly associated with birth weight at a statistical threshold of 5E-8 (representing a conservative Bonferroni correction for approximately one million independent hypotheses, or genetic loci, tested). Interestingly, the genes within a mother that influence her child's birth weight appear distinct from the genes within a child that influence his or her own birth weight. ${ }^{5}$ In this paper, we focus specifically on fetal genetic influences (i.e. the influence of a child's genes on his or her own birth weight), which were shown to explain over four times the amount of variation in birth weight than maternal genetic influences. However, because GWAS are correlational in nature, the extent to which the identified genetic associations are driven by the causal effect of genes versus non-genetic processes (e.g. dynastic effects (Kong et al. 2018; Trejo and Domingue

\footnotetext{
${ }^{5}$ The deattenuated correlation between the vector of allelic weights (i.e. $\left.\vec{\alpha}\right)$ for the fetal effects and the maternal effects, known as the genetic correlation $\left(\rho_{g}\right)$, is quite low $(\sim 0.1)$.
} 
2019) and population stratification (Novembre et al. 2008; Abdellaoui et al. 2019)) remains unclear.

It has become standard practice for researchers to summarize discoveries from GWAS, which rely heavily on very large, cross-sectional samples of individuals and coarse survey measures, as a polygenic score in order to more thoroughly explore the findings in rich, longitudinal studies (Belsky and Harden 2019). Social scientists have already begun unpacking GWAS discoveries for the genetic factors associated with education (Papageorge and Thom 2020; Barth, Papageorge, and Thom 2020; Belsky et al. 2018) and incorporating these polygenic scores into the fetal origins literature (Conley, Sotoudeh, and Laidley 2019). However, few studies, if any, have used polygenic scores to follow up on the recent birth weight GWAS findings. We view this paper as an early step in understanding how genetic influences on birth weight factor into the models of health, social and cognitive development, and human capital formation studied by social scientists.

\section{Data}

We utilize two longitudinal data sources in this paper: The Born in Bradford cohort and The Wisconsin Longitudinal Study. By analyzing both cohorts, we can test a wider range of hypotheses and increase the robustness of our findings. We provide information about each study below.

\section{3a. Born in Bradford}

The Born in Bradford cohort (BiB) is a longitudinal multi-ethnic birth cohort study of the impact of environmental, psychological and genetic factors on maternal and child mental and 
physical health (Wright et al. 2013). The full BiB cohort consists of $\sim 12,500$ women recruited from 2007 to 2010 across $\sim 13,800$ pregnancies, which resulted in $\sim 13,900$ children. White British and Pakistani mothers in roughly equal parts comprise the bulk of the BiB cohort (i.e. 95\%), with the remaining 5\% identifying as other ethnic backgrounds.

At birth, genetic samples were assayed for a subsample of children whose parents consented. Children were genotyped using Illumina HumanCore Exome 12 and 24 BeadChip arrays (Version 1/1.1; Illumina) with umbilical cord blood samples. Healthcare records were collected for mothers and educational records continue to be regularly collected for children. A strength of $\mathrm{BiB}$ for our purposes is that it contains rich clinical and administrative measures which begin when the child is in utero and continue through primary school.

\section{3b. Wisconsin Longitudinal Study}

The Wisconsin Longitudinal Study (WLS) is a survey based on a 1/3 sample of all 1957 Wisconsin high school graduates $(\sim 10,000)$ and a randomly-selected sibling of these graduates (Herd, Carr, and Roan 2014). The graduate respondents were originally empaneled with an inperson questionnaire at age 18 in 1957 , which was followed with data collection at ages 25,36 , 54, 65, and finally 72 in 2012. The WLS includes a wide range of administrative and prospectively collected data from adolescence through adulthood. Early life conditions, like birth weight, were self-reported retrospectively. Beginning in 2008, genetic samples were assayed from saliva for a subsample of consenting WLS graduates and siblings. Genotyping was performed using the Illumina HumanOmniExpress 24 BeadChip arrays (Version 1/1.1; Illumina). Strengths of the WLS particular to our analyses include its large sample of genotyped 
sibling pairs as well as its social and cognitive developmental outcomes from across the life course.

\section{3c. Sample Restriction}

Ancestral diversity raises challenges for the study of human genetics. While there has been recent progress in GWAS in combining individuals of diverse ancestries to identify associations between specific genetic loci and outcomes (Wojcik et al. 2019), diverse ancestries continue to complicate analyses the use genome-wide summary measures such as polygenic scores (Duncan et al. 2019; Martin et al. 2017). For this reason, along with the fact that the GWAS results we use to construct our polygenic scores are derived from individuals of only European ancestries (Warrington et al. 2019), we restrict our BiB and WLS analytic samples to only individuals for whom we have valid genetic data and are of European ancestries (as identified via principal components analysis). This restriction has very different implications for our two samples: WLS respondents are overwhelmingly of European ancestries and, therefore, very few of the respondents are excluded on the basis of ancestry; alternatively, in BiB, we are forced to drop roughly half of the sample of individuals for whom we have valid genetic data (these excluded individuals are largely of South Asian ancestries).

\section{3d. Polygenic Scores}

To calculate polygenic scores, we matched individual genotypes from BiB and the WLS with the most recent GWAS results for birth weight reported by the Early Growth Genomics Consortium (Warrington et al. 2019). Neither BiB nor the WLS were used in the GWAS of birth weight so there is no concern of the polygenic score mechanically predicting outcomes. For each 
genotype, we counted the number of birth weight-associated alleles $(0,1$, or 2$)$, multiplied this count by the effect size estimated in the original GWAS, and then summed weighted counts across all genotypes to calculate each participants' polygenic score. All matched SNPs were used to compute polygenic scores, irrespective of the nominal significance of their association with birth weight. These genome-wide analyses were performed using Plink (Chang et al. 2015). Polygenic scores were then normalized to be mean 0 and standard deviation 1 within each sample.

\section{3e. Non-Genetic Measures}

In addition to polygenic scores, we employ a variety of measures of social and cognitive development at various stages of the life course and prenatal characteristics that may moderate the influence of the polygenic score. Table 1 provides summary statistics for all the measures used in our study.

[Insert Table 1 Here]

See Sections A1 and A2 of the appendix for more details on the non-genetic measures used in this study.

\section{Methods}

4a. The Polygenic Score, Birth Outcomes, and Downstream Development 
To test whether an individual's birth weight polygenic score is associated with a variety of outcomes (e.g. birth weight, other neonatal characteristics, and social and cognitive development later in life), we fit models of the following form for individual $i$ in family $j:^{6}$

$$
Y_{i j}=\hat{\beta}_{0}+\hat{\beta}_{1} \widehat{P G} S_{i j}+\boldsymbol{X}_{i j} \widehat{\boldsymbol{\Theta}}+\epsilon_{i j}
$$

$Y_{i j}$ : Outcome of individual $i$ in family $j$

$\widehat{P G} S_{i j}$ : Birth weight polygenic score for individual $i$ in family $j$

In all of our analyses using polygenic scores, we follow the genetics literature and control for sex, age, ${ }^{7}$ and the first ten principal components of European ancestries genotype to help account for population stratification and, therefore, increase the robustness of our findings (Price et al. 2006).

Nonetheless, an emerging body of evidence suggests that merely controlling for principal components is insufficient to fully reduce environmental confounding due to population stratification and dynastic effects (Zaidi and Mathieson 2020; Berg et al. 2019; Mostafavi et al. 2020; Trejo and Domingue 2019; Kong et al. 2018). Fortunately, conditional on parental genotype, child genotype is randomly assigned through a process known as genetic recombination (Conley and Fletcher 2017). Thus, family fixed effects regression models that compare genetic differences in siblings to phenotypic differences in siblings have a causal

\footnotetext{
${ }^{6}$ There are a small number of sibling pairs in BiB but most respondents will be the only member of their family.

${ }^{7}$ In $\mathrm{BiB}$, we do not observe child's birth date and are therefore unable to control for age in our regression models. However, unlike the WLS, all individuals in BiB were born in the same few year span and are therefore approximately the same age.
} 
interpretation (Rietveld et al. 2014; Domingue et al. 2015; Lee et al. 2018; Belsky et al. 2018; Trejo and Domingue 2019). To that end, in order to test whether the polygenic score indexes a causal effect between an individual's genes and their outcomes, we fit within family models of the following form for individual $i$ in family $j$ using a subsample of sibling pairs in the WLS:

$$
Y_{i j}=\hat{\gamma}_{j}+\hat{\beta}_{1} \widehat{P G} S_{i j}+\boldsymbol{X}_{i j} \widehat{\boldsymbol{\Theta}}+\epsilon_{i j}
$$

$\hat{\gamma}_{j}$ : Family $j$ fixed effects

\section{4b. Testing for Gene-Environment Interactions}

Another key question is whether environmental factors may moderate the relationship between child polygenic score and birth weight. To test for gene-environment interactions, we fit the model for individual $i$ in family $j$ for multiple candidate prenatal environmental features:

$$
Y_{i j}=\hat{\beta}_{0}+\hat{\beta}_{1} \widehat{P G S} S_{i j}+\hat{\beta}_{2} E_{i j}+\hat{\beta}_{3}\left(\widehat{P G S} S_{j} E_{i j}\right)+\boldsymbol{X}_{i j} \widehat{\boldsymbol{\Theta}}+\epsilon_{i j}
$$

$E_{i j}$ : Environmental moderator for individual $i$ in family $j$

Specifically, we test three candidate environmental modifiers in BiB: maternal socioeconomic status, maternal body mass index, and gestational length. Both maternal socioeconomic status and maternal body mass index were measured at 26-28 weeks gestation. We selected socioeconomic status (Aizer and Currie 2014; Conley and Bennett 2000) and body mass index (Yu et al. 2013) because of their well-documented large main effects on birth weight and chose gestational age because of past research using twins that has documented its role in modifying 
genetic influences on neonatal health (Conley, Strully, and Bennett 2006). A statistically

significant $\hat{\beta}_{3}$ point estimate in Equation $4 \mathrm{~b} . \mathrm{i}$ indicates that the change in the conditional expectation associated with a change in each prenatal environment on birth weight depends on a child's genetics.

\section{Results}

\section{5a. The Polygenic Score Predicts Birth Weight}

Our results regarding the relationship between the birth weight polygenic score and observed birth weight, as well as related birth outcomes, are displayed in Table 2.

[Insert Table 2 Here]

Panel A of Table 2 shows the relationship between the birth weight polygenic score and birth weight in both $\mathrm{BiB}$ and the WLS. Model $\mathrm{A} 1$ shows that, in $\mathrm{BiB}$, a one standard deviation increase in the birth weight polygenic score is associated with a 91 gram $(\mathrm{P}<.001)$ increase in birth weight. In the WLS, we see similar results; Model A2 shows that a one standard deviation increase in the birth weight polygenic score is associated with a 102 gram $(\mathrm{P}<.001)$ increase in birth weight. When we include family fixed effects in Model 3 and leverage only genetic differences among siblings, the coefficient attenuates slightly to 88 grams $(\mathrm{P}<.005)$ but remains highly statistically significant. In Panel A of Table A1 in appendix, we replicate the models in Panel A of Table 2 instead using the natural log of birthweight as our outcome variable and yield comparable results. In these models, a one standard deviation increase in the birth weight polygenic score is associated with a $2.5-3 \%$ increase in birth weight. 
Panel B of Table 2 shows the relationship between the birth weight polygenic score and other birth characteristics in $\mathrm{BiB}$. The birth weight polygenic score is negatively associated with being small for gestational length and positively associated with being large for gestational age. Models B2 and B3 show that one standard deviation increase in the birth weight polygenic score is associated with a 3 percentage point $(\mathrm{P}<.001)$, roughly $38 \%$, decrease in the probability of being small for gestational length and a 3 percentage point, roughly $25 \%$, increase the probability of being largely for gestational age. Perhaps surprisingly, Models B1, B4, and B5 show that the birth weight polygenic score is largely unrelated to gestational length and both 1-minute and 5minute postnatal AGPAR scores.

\section{5b. The Polygenic Score Predicts Downstream Outcomes}

We now turn our attention to our results regarding the relationship between the birth weight polygenic score and social and cognitive developmental outcomes, displayed in Table 3.

[Insert Table 3 Here]

In BiB (Panel A), Models A1, A2, and A3 show that a one standard deviation increase in the polygenic score is associated with a 0.04 standard deviation $(\mathrm{P}<.1)$ increase in teacher-rated academic and socioemotional development at age 6 , a 0.06 standard deviation $(\mathrm{P}<.05)$ increase in academic achievement at age 8 , and a 1.8 percentage point $(\mathrm{P}<.05)$, roughly $10 \%$, decrease in the likelihood of having a documented special educational need.

In the WLS (Panel B), we see a similar story. Models B1, B3, and B5 show that a one standard deviation increase in the polygenic score is associated with a .03 standard deviation 
$(\mathrm{P}<.01)$ increase in cognitive ability at age 16 , a .08 year $(\mathrm{p}<.01)$ increase in educational attainment at age 40 , and a 1.4 percentile $(\mathrm{p}<.01)$ increase in high school rank at age 17 . When we include family fixed effects in Models 2 and 4 and compare only differences between siblings ${ }^{8}$ the coefficients on cognitive ability $(.04 ; \mathrm{P}<.1)$ and educational attainment $(.13 ; \mathrm{P}<.05)$ grow in magnitude and remain statistically significant (though the coefficient on cognitive ability is now only significant at a $10 \%$ level). Figure 2 graphically displays the results from Models 1 through 4 in the WLS using binned scatterplots.

\section{[Insert Figure 2 Here]}

\section{5c. Gene-Environment Interactions}

Finally, we turn to our results regarding gene-environment interactions for birth weight, which are displayed in Table 4.

\section{[Insert Table 4 Here]}

Using $\mathrm{BiB}$, we find evidence that the change in birth weight associated with a given change in the polygenic score varies as a function of maternal body mass index and gestation length but not maternal socioeconomic status. Models 1, 2, and 3 show that the coefficients on the interaction terms between the birth weight polygenic score and maternal socioeconomic status (Model 1), maternal body mass index (Model 2), and gestational length (Model 3) are 24 grams $(\mathrm{P}<.1), 6$ grams $(\mathrm{P}<.005)$, and 14 grams $(\mathrm{P}<.05)$, respectively.

\footnotetext{
${ }^{8}$ High school rank was collected only for WLS graduates (and not randomly selected siblings), so a within family analysis for this outcome is not possible.
} 
Figure 3 displays graphically our gene-environment interaction results for birth weight. The plots are generated using augmented versions Models 1, 2, and 3 where the environmental variables are coarsened at their median value into a dichotomous indicator (see Table A2 in the appendix for the results from these regressions).

\section{[Insert Figure 3 Here]}

It may be that the observed GxE results are an artifact of changes to the scaling of the outcome distribution (Domingue et al. 2020). If the distribution of birth weight shifts variance across environments, then a significant $\beta_{3}$ may arise mechanically as a byproduct of the more general increase in variation. For this reason, in Panel B of Table A1 in the appendix, we replicate our GxE analyses using the natural log of birth weight. While the results for maternal $\mathrm{BMI}$ are robust to this $\log$ transformation, we no longer observe evidence for GxE in the case of gestational length.

In Table A3 in the appendix, we rerun our gene-environment interactions models with our social and cognitive developmental measures as outcomes instead of birth weight. We find little evidence that the moderation of the relationship between the birth weight polygenic score and observed birth weight translates into moderation of the relationship between the birth weight polygenic score and social and cognitive outcomes. However, we may simply lack the statistical power to adequately explore this question.

\section{Discussion}

\section{6a. Genetic Influences on Birth Weight}


Using data from two birth cohorts, separated by over 3,000 miles and 70 years, we find that recent genetic discoveries, as summarized by a polygenic score, are robustly associated with birth weight. Sibling comparisons show that this association represents, in large part, a causal effect of an individual's genes on his or her birth weight. Our results suggest that, in the case of birth weight, recent GWAS have identified a relatively accurate, albeit noisy, map linking an individual's DNA sequence to their genetic predisposition to weigh more at birth. However, the polygenic score fails to index an individual's genetic predisposition for other relevant birth characteristics, such as gestational length and APGAR score.

These findings are relevant even to scholars whose research interests primarily center on environmental variation in birth weight. As GWAS sample sizes increase over time and in turn polygenic scores become more accurate and predictive, a birth weight polygenic score may prove useful in removing confounding genetic influences on birth weight and isolating the effect of environmental exposures. For example, residualizing observed birth weight on an individual's polygenic score may reduce bias and increasing precision in research examining the environmental factors that shape prenatal development. The polygenic score for birth weight may also be useful a useful characteristic when testing for antenatal selection and/or changes in fecundity across place and time, which have been shown to be a source of bias in studies of birth outcomes (Nobles and Hamoudi 2019). Finally, the polygenic score for birth weight may be a useful tool in unpacking heterogenous responses (Conley and Fletcher 2017) to environmental exposures during the prenatal period.

Our analyses also help elucidate the causal model that connects birth weight to healthy development later in life. We find that the genetics of birth weight correlate with a constellation of valued downstream outcomes. The polygenic score predicts socioemotional and cognitive 
development starting in childhood and into adulthood. In $\mathrm{BiB}$, children with higher birth weight polygenic scores have increased teacher academic and socioemotional evaluations at age 6, increased academic achievement at age 8 , and are less likely to be categorized as having a special educational need in primary school. In the WLS, individuals with higher birth weight polygenic scores have increased cognitive ability at age 16 , higher class rank in high school, and more years of schooling at age 40. The family fixed effects models in the WLS that compare siblings yield similar results to the between family models, lending the associations between the polygenic score and developmental outcomes a causal interpretation. We therefor conclude that the genetics underpinning of birth weight are causally related an individual's social and cognitive outcomes later in life.

To our knowledge, this is the first evidence that genetic sources of variation in birth weight, like environmental sources, affect other valued life course outcomes. This fact supports the hypothesis that birth weight is intimately related to healthy prenatal development more broadly - evidence consistent with the causal model presented in Panel B of Figure 1. If, on the other hand, birth weight acted solely as an index prenatal environmental exposures that affect both birth weight and healthy prenatal development (i.e. Panel A of Figure 1), we would likely not expect genetic sources of variation in birth weight to translate into downstream social and cognitive outcomes.

Our analyses also provide preliminary support for the idea that an individual's genetic predisposition for birth weight is not fixed but instead interacts with prenatal environmental factors. We find suggestive evidence of gene-environment interactions between the birth weight polygenic score and both maternal body mass index and gestational length. For babies born to mothers with high body mass index, an increase in polygenic score is associated with a larger 
increase in birth weight than it is for babies born to mothers with low body mass index. In addition, for babies that spend a longer time in the womb, an increase in polygenic score is associated with a larger increase in birth weight than it is for babies that spend relatively less time in the womb. However, this gene-environment interaction between polygenic score and gestational length may be driven changes in the overall distribution of birth weight as a function

of gestational length. We find no evidence for gene environment interactions between the polygenic score and maternal socioeconomic status.

Given the methodological challenges associated with using polygenic scores to identify gene-environment interactions in the case of endogenous environments (Domingue et al. 2020), we consider our evidence regarding gene-environment interactions suggestive and deserving further follow up. Nonetheless, these results highlight the importance of jointly considering genetic and environmental factors in understanding the determinants of birth weight and prenatal development more broadly. Curiously, while we observe moderation of the relationship between the birth weight polygenic score and birth weight, we see little evidence for moderation of the relationship between the birth weight polygenic score and downstream developmental outcomes. Thus, properly interpreting the social and health implications of an individual's birth weight may require combining information on both prenatal exposures and the biological characteristics of the child.

\section{6b. Limitations and Interpretational Challenges}

Given the fraught history of discourses regarding individual genetic differences in human health and abilities (Martschenko, Trejo, and Domingue 2019), we hope to be explicit about the 
limitations of the current state of the genetics literature and the resulting interpretational caveats for our analyses using the birth weight polygenic score.

First, the GWAS from which we derive our allelic weights are associational in nature. Thus, our polygenic scores associations do not necessarily capture only the causal effect of an individual's genes. GWAS are at risk for confounding from omitted variables such as population stratification and genetic nurture. Population stratification refers to the fact that an individual's genome contains fine-grained information about their place in the intricate structure of a population (Hamer and Sirota 2000; Novembre et al. 2008; Mostafavi et al. 2020; Abdellaoui et al. 2019); thus, GWAS for complex traits may partly identify genes related to confounding environmental variables such as ancestry, geography, or even socioeconomic status. Genetic nurture refers to the effect of parental genetics on their child's outcome (Kong et al. 2018). For example, a mother's genes affect the birth weight of her child (and a mother's genes are mechanically correlated with her child's genes). Fortunately, it has been shown that the withinfamily polygenic score analyses that we conduct in the WLS can suffer only bias towards zero from between-family confounding, such as population stratification and genetic nurture (Trejo and Domingue 2019).

Second, for the reasons described in Section 3b, we restrict our sample to children of European ancestries. Although we recognize the importance of research in more diverse samples, we can only responsibly move as quickly as the developments in human genomics. We therefore consider our analysis to be an initial pass at considering the role of the genes in the production of birth weight and human development. Because both environmental exposures (Bertrand and Mullainathan 2004; Fryer 2019; Lu and Halfon 2003) and the distribution of birth weight (Aizer 
and Currie 2014) vary across ancestries, we emphasize that the results in this paper apply only to individuals of European ancestries.

Third, our polygenic scores contain large amounts of measurement error (i.e. $\mu_{i}$ in equation $2 \mathrm{~b} . \mathrm{iii}$ is large). It is therefore impossible to use the current birth weight polygenic score to draw conclusions about the relative importance of genetic characteristics versus environmental factors for birth weight and downstream human capital accumulation. Nonetheless, as others have pointed out (Papageorge and Thom 2020), polygenic scores allow researchers to test for associations and interaction effects. As sample sizes for genetic discovery increase and geneticists hone their statistical methodologies, the amount of measurement error in polygenic scores will approach zero. At that point, polygenic scores may become even more useful tools for controlling for genetic factors in studies of the environment and for decomposing the variance between genetic and environmental factors.

\section{6c. Future Directions}

We hope that this paper represents the beginning of a broader effort to understand genetic influences on prenatal development. Our work documents that the genetics of birth weight contribute alongside and likely modify environmental exposures in shaping birth weight, but the precise mechanisms remain unclear. Future work should prioritize uncovering the processes through which the birth weight polygenic affects both birth weight and downstream social and cognitive outcomes.

Further, in this paper, we restricted our focus to understanding the direct genetic effects of an individual's genes on his or her own birth weight. However, birth weight is a strong candidate for the study of indirect genetic effects (Domingue and Belsky 2017; Kong et al. 2018; 
Armstrong-Carter et al. 2020) using polygenic scores - the influence of maternal and paternal genetics on child birth weight merits future attention. Finally, birth weight is just one measure of prenatal development; future researchers should work to build an increased understanding of the genetic underpinnings of other aspects of prenatal development besides birth weight (Conti et al. 2020).

We have provided evidence that genetic sources of variation in birth weight, long ignored, index meaningful differences in experiences prenatally through adulthood, both between and within families. The continued integration of genetic information into social scientific models of human health and behavior will serve to further our understanding of the complex ways in which biological and social factors combine to shape valued outcomes across the life course. 


\section{Appendix}

\section{A1. Non-Genetic Measures: BiB}

Birth Weight: Birth weight in grams was clinically assessed by hospital staff at delivery. Maternal Body Mass Index: Maternal body mass index was calculated using height and weight measurements that were directly assessed by medical staff at 26-28 weeks gestation.

Maternal Socioeconomic Status: Maternal socioeconomic status is measured by the first principle component of five maternal characteristics measured at 26-28 weeks gestation: educational attainment, receipt of mean-tested governmental benefits, neighborhood Index of Multiple Deprivation, partner cohabitation, and home ownership.

Gestational Length: Child gestational age was obtained from maternal clinical pregnancy records.

Size for Gestational Length: A child was deemed small or large for gestational length if his or her birth weight was below the 10th percentile or above the 90th percentile, respectively, of the birth weight distribution for babies of the same gestational length.

APGAR Scores: One-minute postnatal and five-minute postnatal APGAR scores were clinically assessed by hospital staff at delivery.

Teacher-Rated Academic and Socioemotional Development: The Early Years Foundation Stage Profile is a teacher-led observational assessment conducted towards the end of the child's first year at school when they are roughly six years old. The profile measures children's attainment in seven main areas of learning: communication and language; expressive arts and design; literacy, mathematics; physical development; personal, socio and emotional development and understanding the world. Teachers completed the assessment for each child based on their 
knowledge and observations of that child. Subscales scores were summed and standardized within sample to be mean 0 and standard deviation 1.

Academic Achievement: The Key Stage 1 Assessment is a standardized test administered at the end of a child's third year in school when the children are typically eight years old. The Key Stage 1 Assessment includes math, reading and science subscales. Subscale scores were summed and standardized within sample to be mean 0 and standard deviation 1 .

Special Educational Need: A binary indicator for special educational need was extracted from the child's educational records when they were eight years old.

\section{A2. Non-Genetic Measures: WLS}

Birth Weight: Birth weight in pounds and ounces was collected from survey responses when respondents were in their 60s.

Cognitive Ability: Cognitive ability was assessed using the Henmon-Nelson Test of Mental Ability, which was administered to WLS participants during high school. It is a 30minute test consisting of 90 items including vocabulary, sentence completion, disarranged sentences, classification, logical selection, series completion, directions, analogies, anagrams, proverb interpretation, and arithmetic problems.

Educational Attainment: Years of schooling collected from survey responses when respondents were in their 40s.

High School Rank: High school percentile rank was calculated using each respondent's GPA compared to the GPA of other students at his or her school. GPA information was collected from administrative educational records. This variable exists only for original WLS graduates and not for their randomly selected sibling. 


\section{References}

Abdellaoui, Abdel, David Hugh-Jones, Loic Yengo, Kathryn E. Kemper, Michel G. Nivard, Laura Veul, Yan Holtz, et al. 2019. "Genetic Correlates of Social Stratification in Great Britain.” Nature Human Behaviour. https://doi.org/10.1038/s41562-019-0757-5.

Aizer, Anna, and Janet Currie. 2014. "The Intergenerational Transmission of Inequality: Maternal Disadvantage and Health at Birth." Science. https://doi.org/10.1126/science.1251872.

Almond, Douglas, Ky Chay, and Ds Lee. 2005. "The Costs of Low Birth Weight." The Quarterly Journal of Economics 120 (3): 1031-83. https://doi.org/10.1162/003355305774268228.

Almond, Douglas, and Janet Currie. 2011. Human Capital Development before Age Five. Handbook of Labor Economics. Vol. 4. Elsevier B.V. https://doi.org/10.1016/S01697218(11)02413-0.

Almond, Douglas, Janet Currie, and Valentina Duque. 2018. "Childhood Circumstances and Adult Outcomes: Act II.” Journal of Economic Literature. https://doi.org/10.1257/jel.20171164.

Armstrong-Carter, Emma, Sam Trejo, Liam Hill, Kirsty Crossley, Dan Mason, and Benjamin W. Domingue. 2019. "The Earliest Origins of Genetic Nurture: Prenatal Environment Mediates the Association Between Maternal Genetics and Child Development." Psyarxiv. https://doi.org/10.22201/fq.18708404e.2004.3.66178.

Armstrong-Carter, Emma, Sam Trejo, Liam J.B. Hill, Kirsty L. Crossley, Dan Mason, and Benjamin W. Domingue. 2020. "The Earliest Origins of Genetic Nurture: The Prenatal Environment Mediates the Association Between Maternal Genetics and Child Development." Psychological Science. https://doi.org/10.1177/0956797620917209.

Barcellos, Silvia H., Leandro S. Carvalho, and Patrick Turley. 2018. "Education Can Reduce Health Differences Related to Genetic Risk of Obesity." Proceedings of the National Academy of Sciences. https://doi.org/10.1007/BF00871674.

Barker, D. 1990. "The Fetal and Infant Origins of Adult Disease." BMJ: British Medical Journal 301 (156): 1111. https://doi.org/10.1136/bmj.301.6761.1111. - 1995. "The Fetal and Infant Origins of Disease." European Journal of Clinical Investigation. https://doi.org/10.1111/j.1365-2362.1995.tb01730.x.

Barth, Daniel, Nicholas W. Papageorge, and Kevin Thom. 2020. "Genetic Endowments and Wealth Inequality.” Journal of Political Economy. https://doi.org/10.1086/705415.

Beauchamp, Jonathan P, David Cesarini, Magnus Johannesson, Matthijs J. H. M van der Loos, Philipp D Koellinger, Patrick J. F Groenen, James H Fowler, J. Niels Rosenquist, A. Roy Thurik, and Nicholas A Christakis. 2011. "Molecular Genetics and Economics." Journal of Economic Perspectives. https://doi.org/10.1257/jep.25.4.57.

Behrman, Jere R, and Mark R Rosenzweig. 2004. "Returns to Birthweight." Review of Economics and Statistics 86 (2): 586-601. https://doi.org/10.1162/003465304323031139.

Belsky, Daniel W., Benjamin W. Domingue, Robbee Wedow, Louise Arseneault, Jason D. Boardman, Avshalom Caspi, Dalton Conley, et al. 2018. "Genetic Analysis of Social-Class Mobility in Five Longitudinal Studies.” Proceedings of the National Academy of Sciences. https://doi.org/10.1073/pnas.1801238115.

Belsky, Daniel W., and K. Paige Harden. 2019. "Phenotypic Annotation: Using Polygenic Scores to Translate Discoveries From Genome-Wide Association Studies From the Top Down." 
Current Directions in Psychological Science. https://doi.org/10.1177/0963721418807729.

Benjamin, Daniel J., David Cesarini, Christopher F. Chabris, Edward L. Glaeser, David I.

Laibson, Vilmundur Guðnason, Tamara B. Harris, et al. 2012. "The Promises and Pitfalls of Genoeconomics." Annual Review of Economics. https://doi.org/10.1146/annureveconomics-080511-110939.

Berg, Jeremy J., Arbel Harpak, Nasa Sinnott-Armstrong, Anja Moltke Joergensen, Hakhamanesh Mostafavi, Yair Field, Evan August Boyle, et al. 2019. "Reduced Signal for Polygenic Adaptation of Height in UK Biobank." ELife. https://doi.org/10.7554/eLife.39725.

Bertrand, M, and S Mullainathan. 2004. "Are Greg and Emily More Employable Then Lakisha and Jamal? A Field Experiment on Labor Market Discrimination.” American Economic Review.

Black, Sandra E., Paul J. Devereux, and Kjell G. Salvanes. 2007. "From the Cradle to the Labor Market? The Effect of Birth Weight on Adult Outcomes." The Quarterly Journal of Economics 122 (February): 409-39. https://doi.org/10.1162/qjec.122.1.409.

Boardman, Jason D., Benjamin W. Domingue, Casey L. Blalock, Brett C. Haberstick, Kathleen Mullan Harris, and Matthew B. McQueen. 2014. "Is the Gene-Environment Interaction Paradigm Relevant to Genome-Wide Studies? The Case of Education and Body Mass Index." Demography. https://doi.org/10.1007/s13524-013-0259-4.

Boardman, Jason D., Daniel A. Powers, Yolanda C. Padilla, and Robert A. Hummer. 2002. "Low Birth Weight, Social Factors, and Developmental Outcomes among Children in the United States" 39 (2): 353-68.

Boyle, Evan A., Yang I. Li, and Jonathan K. Pritchard. 2017. "An Expanded View of Complex Traits: From Polygenic to Omnigenic.” Cell. https://doi.org/10.1016/j.cell.2017.05.038.

Brumpton, Ben, Eleanor Sanderson, Karl Heilbron, Fernando Pires Hartwig, Sean Harrison, Gunnhild Åberge Vie, Yoonsu Cho, et al. 2020. “Avoiding Dynastic, Assortative Mating, and Population Stratification Biases in Mendelian Randomization through within-Family Analyses." Nature Communications. https://doi.org/10.1038/s41467-020-17117-4.

Chabris, C. F., J. J. Lee, D. Cesarini, D. J. Benjamin, and D. I. Laibson. 2015. "The Fourth Law of Behavior Genetics." Current Directions in Psychological Science 24 (4): 304-12. https://doi.org/10.1177/0963721415580430.

Chang, Christopher C., Carson C. Chow, Laurent C.A.M. Tellier, Shashaank Vattikuti, Shaun M. Purcell, and James J. Lee. 2015. "Second-Generation PLINK: Rising to the Challenge of Larger and Richer Datasets." GigaScience. https://doi.org/10.1186/s13742-015-0047-8.

Conley, Dalton, and Neil G. Bennett. 2000. "Is Biology Destiny? Birth Weight and Life Chances.” American Sociological Review 65 (3): 458. https://doi.org/10.2307/2657467.

Conley, Dalton, and Jason Fletcher. 2017. The Genome Factor What the Social Genomics Revolution Reveals about Ourselves, Our History, and the Future.

Conley, Dalton, Ramina Sotoudeh, and Thomas Laidley. 2019. "Birth Weight and Development: Bias or Heterogeneity by Polygenic Risk Factors?” Population Research and Policy Review. https://doi.org/10.1007/s11113-019-09559-6.

Conley, Dalton, Kate W. Strully, and Neil G. Bennett. 2006. "Twin Differences in Birth Weight: The Effects of Genotype and Prenatal Environment on Neonatal and Post-Neonatal Mortality." Economics and Human Biology. https://doi.org/10.1016/j.ehb.2005.12.001.

Conti, Gabriella, Keith Godfrey, Gabriella Conti, Sarah Crozier, and Hazel Inskip. 2020. "Beyond Birthweight: The Origins of Human Capital." IZA Working Paper, no. 13296. Domingue, Benjamin W., and Daniel W. Belsky. 2017. "The Social Genome: Current Findings 
and Implications for the Study of Human Genetics." PLoS Genetics 13 (3).

https://doi.org/10.1371/journal.pgen.1006615.

Domingue, Benjamin W., Sam Trejo, Emma Armstrong-Carter, and Elliot Tucker-Drob. 2020. "Interactions between Polygenic Scores and Environments: A Discussion of Methodological and Conceptual Problems." SocArXiv.

Domingue, Benjamin W, Daniel W Belsky, Dalton Conley, Kathleen Mullan Harris, and Jason D Boardman. 2015. "Polygenic Influence on Educational Attainment: New Evidence From the National Longitudinal Study of Adolescent to Adult Health.” AERA Open 1 (3): 1-13. https://doi.org/10.1177/2332858415599972.

Dudbridge, Frank. 2013. "Power and Predictive Accuracy of Polygenic Risk Scores." PLoS Genetics 9 (3). https://doi.org/10.1371/journal.pgen.1003348.

Duncan, L., H. Shen, B. Gelaye, J. Meijsen, K. Ressler, M. Feldman, R. Peterson, and B. Domingue. 2019. "Analysis of Polygenic Risk Score Usage and Performance in Diverse Human Populations.” Nature Communications. https://doi.org/10.1038/s41467-019-111120 .

Figlio, David, Jonathan Guryan, Krzysztof Karbownik, and Jeffrey Roth. 2014. "The Effects of Poor Neonatal Health on Children's Cognitive Development?" American Economic Review. https://doi.org/10.1257/aer.104.12.3921.

Fryer, Roland G. 2019. "An Empirical Analysis of Racial Differences in Police Use of Force." Journal of Political Economy. https://doi.org/10.1086/701423.

Giri, Ayush, Jacklyn N Hellwege, Jacob M Keaton, Jihwan Park, Chengxiang Qiu, Helen R Warren, Eric S Torstenson, et al. 2019. "Trans-Ethnic Association Study of Blood Pressure Determinants in over 750,000 Individuals." Nature Genetics 51 (1): 51.

Hamer, D. H., and L. Sirota. 2000. "Beware the Chopsticks Gene." Molecular Psychiatry. https://doi.org/10.1038/sj.mp.4000662.

Harden, K. Paige, and Philipp D. Koellinger. 2020. "Using Genetics for Social Science." Nature Human Behaviour. https://doi.org/10.1038/s41562-020-0862-5.

Herd, Pamela, Deborah Carr, and Carol Roan. 2014. "Cohort Profile: Wisconsin Longitudinal Study (WLS).” International Journal of Epidemiology 43 (1): 34-41. https://doi.org/10.1093/ije/dys194.

Hyde, Craig L., Michael W. Nagle, Chao Tian, Xing Chen, Sara A. Paciga, Jens R. Wendland, Joyce Y. Tung, David A. Hinds, Roy H. Perlis, and Ashley R. Winslow. 2016.

"Identification of 15 Genetic Loci Associated with Risk of Major Depression in Individuals of European Descent." Nature Genetics. https://doi.org/10.1038/ng.3623.

Karlsson Linnér, Richard, Pietro Biroli, Edward Kong, S. Fleur W. Meddens, Robbee Wedow, Mark Alan Fontana, Maël Lebreton, et al. 2019. "Genome-Wide Association Analyses of Risk Tolerance and Risky Behaviors in over 1 Million Individuals Identify Hundreds of Loci and Shared Genetic Influences.” Nature Genetics. https://doi.org/10.1038/s41588-0180309-3.

Kong, Augustine, Gudmar Thorleifsson, Michael L. Frigge, Bjarni J. Vilhjalmsson, Alexander I. Young, Thorgeir E. Thorgeirsson, Stefania Benonisdottir, et al. 2018. "The Nature of Nurture: Effects of Parental Genotypes.” Science 359 (6374): 424-28. https://doi.org/10.1126/science.aan6877.

Lander, Eric S., Lauren M. Linton, Bruce Birren, Chad Nusbaum, Michael C. Zody, Jennifer Baldwin, Keri Devon, et al. 2001. "Initial Sequencing and Analysis of the Human Genome.” Nature. https://doi.org/10.1038/35057062. 
Lee, James J., Robbee Wedow, Aysu Okbay, Edward Kong, Omeed Maghzian, Meghan Zacher, Tuan Anh Nguyen-Viet, et al. 2018. "Gene Discovery and Polygenic Prediction from a Genome-Wide Association Study of Educational Attainment in 1.1 Million Individuals." Nature Genetics. https://doi.org/10.1038/s41588-018-0147-3.

Lu, Michael C., and Neal Halfon. 2003. "Racial and Ethnic Disparities in Birth Outcomes: A Life-Course Perspective." Maternal and Child Health Journal. https://doi.org/10.1023/A:1022537516969.

Martin, Alicia R., Christopher R. Gignoux, Raymond K. Walters, Genevieve L. Wojcik, Benjamin M. Neale, Simon Gravel, Mark J. Daly, Carlos D. Bustamante, and Eimear E. Kenny. 2017. "Human Demographic History Impacts Genetic Risk Prediction across Diverse Populations.” American Journal of Human Genetics. https://doi.org/10.1016/j.ajhg.2017.03.004.

Martschenko, Daphne, Sam Trejo, and Benjamin W. Domingue. 2019. "Genetics and Education: Recent Developments in the Context of an Ugly History and an Uncertain Future." AERA Open. https://doi.org/10.1177/2332858418810516.

Mills, Melinda C., and Felix C. Tropf. 2020. "Sociology, Genetics, and the Coming of Age of Sociogenomics." Annual Review of Sociology. https://doi.org/10.1146/annurev-soc-121919054756.

Mostafavi, Hakhamanesh, Arbel Harpak, Ipsita Agarwal, Dalton Conley, Jonathan K. Pritchard, and Molly Przeworski. 2020. "Variable Prediction Accuracy of Polygenic Scores within an Ancestry Group.” ELife. https://doi.org/10.7554/eLife.48376.

Nobles, Jenna, and Amar Hamoudi. 2019. "Detecting the Effects of Early-Life Exposures: Why Fecundity Matters." Population Research and Policy Review. https://doi.org/10.1007/s11113-019-09562-x.

Novembre, John, Toby Johnson, Katarzyna Bryc, Zoltán Kutalik, Adam R. Boyko, Adam Auton, Amit Indap, et al. 2008. "Genes Mirror Geography within Europe.” Nature. https://doi.org/10.1038/nature07331.

Okbay, Aysu, Bart M.L. Baselmans, Jan Emmanuel De Neve, Patrick Turley, Michel G. Nivard, Mark Alan Fontana, S. Fleur W. Meddens, et al. 2016. "Genetic Variants Associated with Subjective Well-Being, Depressive Symptoms, and Neuroticism Identified through Genome-Wide Analyses." Nature Genetics. https://doi.org/10.1038/ng.3552.

Oreopoulos, P., M. Stabile, R. Walld, and L. L. Roos. 2008. "Short-, Medium-, and Long-Term Consequences of Poor Infant Health: An Analysis Using Siblings and Twins." Journal of Human Resources 43: 88-138. https://doi.org/10.1353/jhr.2008.0003.

Papageorge, Nicholas W., and Kevin Thom. 2020. "Genes, Education, and Labor Market Outcomes: Evidence from the Health and Retirement Study." Journal of the European Economic Association. https://doi.org/10.1093/jeea/jvz072.

Pearson, Thomas a, and Teri a Manolio. 2008. "How to Interpret a Genome-Wide Association Study." JAMA : The Journal of the American Medical Association 299 (11): 1335-44. https://doi.org/10.1001/jama.299.11.1335.

Polderman, Tinca J C, Beben Benyamin, Christiaan A de Leeuw, Patrick F Sullivan, Arjen van Bochoven, Peter M Visscher, and Danielle Posthuma. 2015. "Meta-Analysis of the Heritability of Human Traits Based on Fifty Years of Twin Studies." Nature Genetics 47 (7): 702-9. https://doi.org/10.1038/ng.3285.

Price, Alkes L., Nick J. Patterson, Robert M. Plenge, Michael E. Weinblatt, Nancy A. Shadick, and David Reich. 2006. "Principal Components Analysis Corrects for Stratification in 
Genome-Wide Association Studies.” Nature Genetics. https://doi.org/10.1038/ng1847.

Rietveld, Cornelius A, Dalton Conley, Nicholas Eriksson, Tõnu Esko, Sarah E Medland, Anna A E Vinkhuyzen, Jian Yang, et al. 2014. "Replicability and Robustness of Genome-WideAssociation Studies for Behavioral Traits.” Psychological Science 25 (11): 1975-86. https://doi.org/10.1177/0956797614545132.

Royer, Heather. 2009. "Separated at Girth: US Twin Estimates of the Effects of Birth Weight." American Economic Journal: Applied Economics. https://doi.org/10.1257/app.1.1.49.

Torche, Florencia, and Ghislaine Echevarría. 2011. "The Effect of Birthweight on Childhood Cognitive Development in a Middle-Income Country." International Journal of Epidemiology 40 (4): 1008-18. https://doi.org/10.1093/ije/dyr030.

Trejo, Sam, and Benjamin W. Domingue. 2019. "Genetic Nature or Genetic Nurture?

Introducing Social Genetic Parameters to Quantify Bias in Polygenic Score Analyses." Biodemography and Social Biology. https://doi.org/10.1080/19485565.2019.1681257.

Victora, Cesar G., Linda Adair, Caroline Fall, Pedro C. Hallal, Reynaldo Martorell, Linda Richter, and Harshpal Singh Sachdev. 2008. "Maternal and Child Undernutrition: Consequences for Adult Health and Human Capital." The Lancet. https://doi.org/10.1016/S0140-6736(07)61692-4.

Visscher, Peter M., Matthew A. Brown, Mark I. McCarthy, and Jian Yang. 2012. "Five Years of GWAS Discovery." American Journal of Human Genetics 90 (1): 7-24. https://doi.org/10.1016/j.ajhg.2011.11.029.

Visscher, Peter M., Naomi R. Wray, Qian Zhang, Pamela Sklar, Mark I. McCarthy, Matthew A. Brown, and Jian Yang. 2017. "10 Years of GWAS Discovery: Biology, Function, and Translation." American Journal of Human Genetics. https://doi.org/10.1016/j.ajhg.2017.06.005.

Warrington, Nicole M., Robin N. Beaumont, Momoko Horikoshi, Felix R. Day, Øyvind Helgeland, Charles Laurin, Jonas Bacelis, et al. 2019. "Maternal and Fetal Genetic Effects on Birth Weight and Their Relevance to Cardio-Metabolic Risk Factors." Nature Genetics. https://doi.org/10.1038/s41588-019-0403-1.

Wojcik, Genevieve L., Mariaelisa Graff, Katherine K. Nishimura, Ran Tao, Jeffrey Haessler, Christopher R. Gignoux, Heather M. Highland, et al. 2019. "Genetic Analyses of Diverse Populations Improves Discovery for Complex Traits.” Nature. https://doi.org/10.1038/s41586-019-1310-4.

Wooldridge, Jeffrey M. 2015. Introductory Econometrics : A Modern Approach. Introductory Econometrics : A Modern Approach.

Wright, John, Neil Small, Pauline Raynor, Derek Tuffnell, Raj Bhopal, Noel Cameron, Lesley Fairley, et al. 2013. "Cohort Profile: The Born in Bradford Multi-Ethnic Family Cohort Study.” International Journal of Epidemiology. https://doi.org/10.1093/ije/dys112.

Yengo, Loic, Julia Sidorenko, Kathryn E. Kemper, Zhili Zheng, Andrew R. Wood, Michael N. Weedon, Timothy M. Frayling, Joel Hirschhorn, Jian Yang, and Peter M. Visscher. 2018. "Meta-Analysis of Genome-Wide Association Studies for Height and Body Mass Index in 700000 Individuals of European Ancestry." Human Molecular Genetics. https://doi.org/10.1093/hmg/ddy271.

Yu, Zhangbin, Shuping Han, Jingai Zhu, Xiaofan Sun, Chenbo Ji, and Xirong Guo. 2013. "Pre-

Pregnancy Body Mass Index in Relation to Infant Birth Weight and Offspring Overweight/Obesity: A Systematic Review and Meta-Analysis." PLoS ONE. https://doi.org/10.1371/journal.pone.0061627. 
Zaidi, Arslan A, and Iain Mathieson. 2020. "Demographic History Impacts Stratification in Polygenic Scores.” BioRxiv. https://doi.org/10.1101/2020.07.20.212530. 


\section{Tables}

Table 1. Summary Statistics.

\begin{tabular}{|c|c|c|c|c|c|c|}
\hline \multirow[t]{2}{*}{ A. Born in Bradford } & \multicolumn{3}{|c|}{ Individual } & & & \\
\hline & Mean & SD & $\mathrm{N}$ & & & \\
\hline Birth Weight Polygenic Score & 0.00 & 1.00 & 2008 & & & \\
\hline Female & 0.48 & 0.50 & 2008 & & & \\
\hline Maternal Body Mass Index & 27.16 & 6.13 & 1741 & & & \\
\hline Family Socioeconomic Status & 0.00 & 1.00 & 1629 & & & \\
\hline Birth Weight (Grams) & 3387.83 & 531.37 & 2007 & & & \\
\hline Gestational Length (Weeks) & 39.68 & 1.61 & 2008 & & & \\
\hline Small for Gestational Length & 0.08 & 0.27 & 1977 & & & \\
\hline Large for Gestational Length & 0.12 & 0.32 & 1977 & & & \\
\hline APGAR (1 Minute) & 8.49 & 1.27 & 2000 & & & \\
\hline APGAR (5 Minute) & 9.08 & 0.58 & 1995 & & & \\
\hline Teacher-Rated Development (Age 6) & 0.00 & 1.00 & 1951 & & & \\
\hline Academic Achievement (Age 8) & 0.00 & 1.00 & 1557 & & & \\
\hline Special Educational Need & 0.17 & 0.38 & 2008 & & & \\
\hline \multirow[t]{2}{*}{ B. Wisconsin Longitudinal Study } & \multicolumn{3}{|c|}{ Individual } & \multicolumn{3}{|c|}{ Sibling Difference } \\
\hline & Mean & SD & $\mathrm{N}$ & Mean & SD & $\mathrm{N}$ \\
\hline Birth Weight Polygenic Score & 0.00 & 1.00 & 8488 & 0.80 & 0.62 & 2050 \\
\hline WLS Graduate & 0.64 & 0.48 & 8488 & 1.00 & 0.00 & 2050 \\
\hline Female & 0.52 & 0.50 & 8488 & 0.51 & 0.50 & 2050 \\
\hline Birth Date (Years) & 1940.07 & 4.28 & 8488 & 5.90 & 4.17 & 2050 \\
\hline Birth Weight (Grams) & 3385.28 & 633.52 & 4934 & 584.14 & 519.68 & 744 \\
\hline Cognitive Ability (Age 16) & 0.00 & 1 & 7993 & 0.89 & 0.70 & 1731 \\
\hline High School Rank (Percentile) & 53.90 & 28.30 & 5073 & - & - & - \\
\hline Years of Schooling & 13.77 & 2.39 & 8350 & 1.91 & 2.00 & 1980 \\
\hline
\end{tabular}

Note. This table uses data from the Born and Bradford cohort and the Wisconsin Longitudinal Study. Additional details about these variables can be found in the appendix. 
Table 2. Neonatal Regressions.

Born in Bradford

A. Birth Weight

Birth Weight Polygenic Score

Family Fixed Effects

Observations

Families

ects

\section{Wisconsin Longitudinal Study}

\begin{tabular}{ccc}
\hline$(1)$ & $(2)$ & $(3)$ \\
\hline $91.44^{* *}$ & $101.5^{* *}$ & $87.68^{* *}$ \\
$(11.96)$ & $(9.316)$ & $(28.47)$ \\
& & $X$ \\
2007 & 4934 & 1488 \\
1930 & 4190 & 744 \\
\hline
\end{tabular}

Born in Bradford

B. Other Birth Characteristics

Gestational Length
(Weeks)

(Weeks)

Birth Weight Polygenic Score

0.00884

(0.0388)

4190

744
(1)

(4)

APGAR

Large for

Small for

Gestational Length

$-0.0334 * *$

(0.00660)

$\begin{array}{ll}2008 & 1977 \\ 1931 & 1907\end{array}$

1977
1907

$* p<.05$

$* * \mathrm{p}<.01$

\begin{tabular}{l} 
Observations \\
Families \\
\hline$+p<.10 \quad * p<.05 \quad * * p<.01$
\end{tabular}

Note. This table uses data from the Born and Bradford cohort and the Wisconsin Longitudinal Study. The outcome variable of all regressions in Panel A is birth weight measured in grams. Regressions in the Born the Bradford cohort control for sex and the first 10 principal components of individual genotype. Regressions in the Wisconsin Longitudinal Study control for a dichotomous indicator for whether an individual is a WLS graduate or randomly selected sibling, birth year, sex, and the first 10 principal components of individual genotype. In $\mathrm{BiB}$, birth weight and other birth characteristics were clinically assessed at birth. In the Wisconsin Longitudinal Study, birth weight was self-reported retrospectively. Robust standard errors are clustered at the family-level. 
Table 3. Social and Cognitive Development Regressions

\begin{tabular}{|c|c|c|c|c|c|}
\hline \multirow[t]{2}{*}{ A. Born in Bradford } & (1) & $(2)$ & (3) & & \\
\hline & $\begin{array}{c}\text { Teacher-Rated } \\
\text { Development }\end{array}$ & $\begin{array}{c}\text { Academic } \\
\text { Achievement }\end{array}$ & $\begin{array}{c}\text { Special } \\
\text { Educational Need }\end{array}$ & & \\
\hline \multirow[t]{2}{*}{ Birth Weight Polygenic Score } & $0.0405+$ & $0.0644^{*}$ & $-0.0180^{*}$ & & \\
\hline & $(0.0228)$ & $(0.0252)$ & $(0.00822)$ & & \\
\hline Observations & 1951 & 1557 & 2008 & & \\
\hline Families & 1878 & 1515 & 1931 & & \\
\hline \multirow[t]{2}{*}{ B. Wisconsin Longitudinal Study } & $(1)$ & $(2)$ & (3) & $(4)$ & (5) \\
\hline & \multicolumn{2}{|c|}{ Cognitive Ability } & \multicolumn{2}{|c|}{ Years of Schooling } & High School Rank \\
\hline \multirow[t]{2}{*}{ Birth Weight Polygenic Score } & $0.0327 * *$ & $0.0434+$ & $0.0761 * *$ & $0.134^{*}$ & $1.139 * *$ \\
\hline & $(0.0116)$ & $(0.0263)$ & $(0.0271)$ & $(0.0602)$ & $(0.380)$ \\
\hline Family Fixed Effects & & $x$ & & $x$ & \\
\hline Observations & 7993 & 3462 & 8350 & 3960 & 5073 \\
\hline Families & 6262 & 1731 & 6370 & 1980 & 5073 \\
\hline
\end{tabular}

$+p<.10 \quad * p<.05 \quad * * p<.01$

Note. This table uses data from the Born and Bradford cohort and the Wisconsin Longitudinal Study. Regressions in the Born the Bradford cohort control for sex and the first 10 principal components of individual genotype. Regressions in the Wisconsin Longitudinal Study control for a dichotomous indicator for whether an individual is a WLS graduate or randomly selected sibling, birth year, sex, and the first 10 principal components of individual genotype. In BiB, teacher-rated development (age 6), academic achievement (age 8), and special educational need were collected from a student's primary school education record. In the Wisconsin Longitudinal Study, cognitive ability was assessed in high school and years of schooling was self-reported at age 40 . Robust standard errors are clustered at the family-level. 
Table 4. GxE Birth Weight Regressions.

Born in Bradford

Birth Weight Polygenic Score

$4.38^{* *}$

(13.16)

$56.58 * *$

(13.42)

Maternal Socioeconomic Status

$24.01+$

Birth Weight Polygenic Score X Family Socioeconomic Status

(13.07)

Maternal Body Mass Index

Gestational Length

Birth Weight Polygenic Score X Gestational Length

Observations
Families

Birth Weight Polygenic Score X Maternal Body Mass Index
$17.95^{* *}$

(2.062)

$6.048 * *$

(2.057)

$(2)$
$85.46^{* *}$
$(12.07)$


$17.95^{* *}$
$(2.062)$
$6.048^{* *}$
$(2.057)$

$90.75^{* *}$

(10.24)

Note. All regressions use data from the Bradford in Bradford cohort and control for sex and the first 10 principal components of individual genotype. The outcome variable of all regressions is birth weight measured in grams. Covariates are centered at their mean. Birth weight and gestational length were clinically assessed at birth. Maternal body mass index was clinically assessed at $\sim 27$ weeks gestation. Maternal SES is the first principal component of five maternal variables: educational attainment, receipt of mean-tested governmental benefits, neighborhood Index of Multiple Deprivation, partner cohabitation, and home ownership. Robust standard errors are clustered at the family-level. 


\section{Figures}

Figure 1

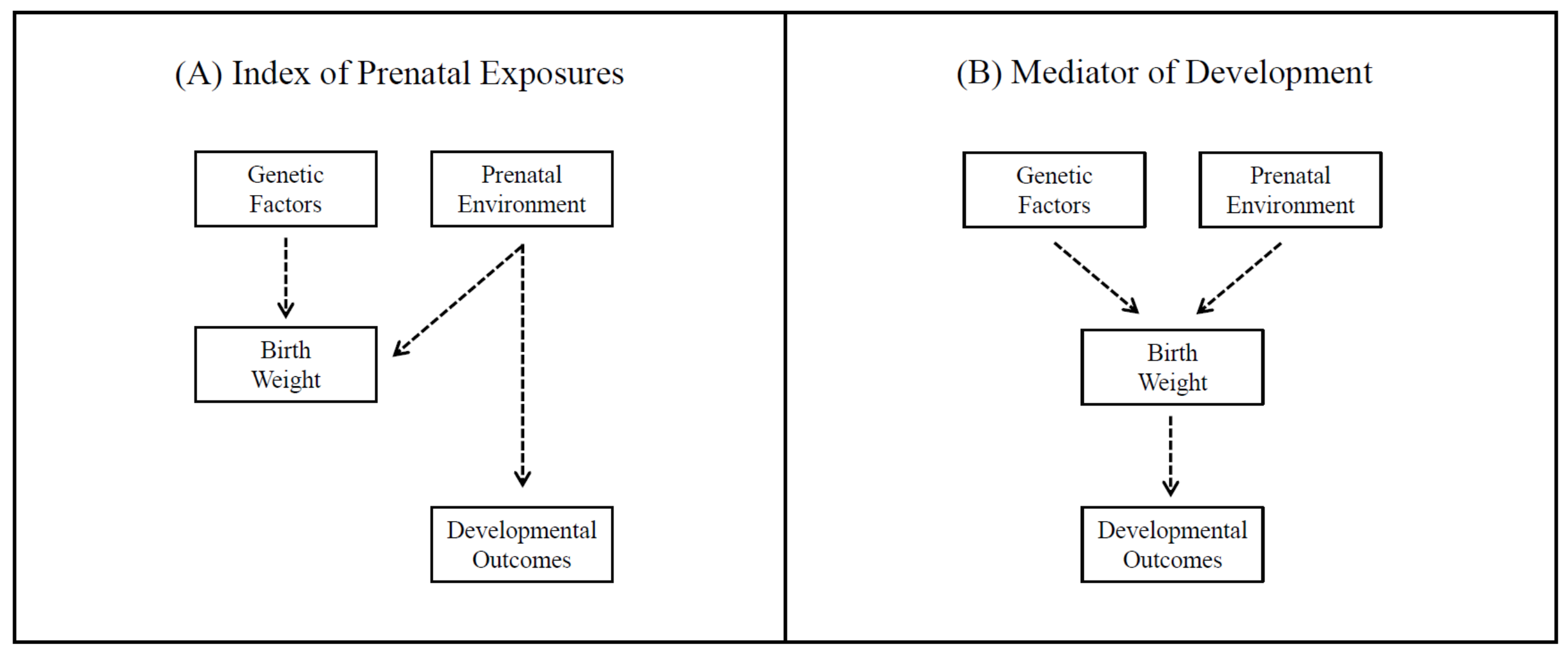

Note. This figure displays two potential causal models linking genes, the prenatal environment, birth weight, and downstream human development. 
Figure 2

\section{Birth Weight Polygenic Score Predicts Cognitive Ability and Educational Attainment}

Between Families

A)

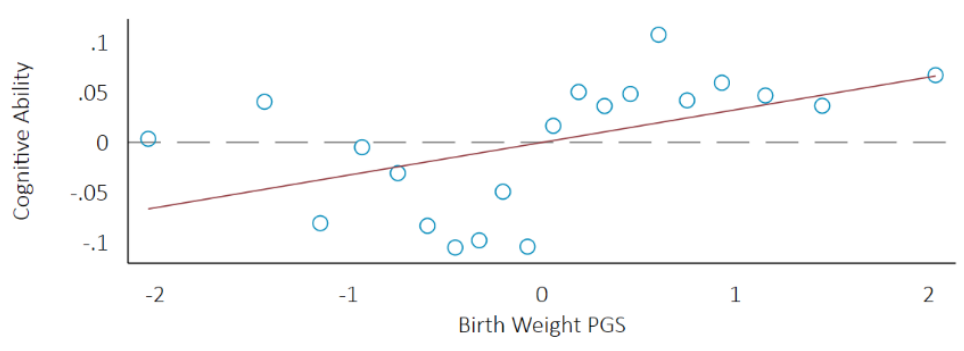

$N=7993$ individuals

C)

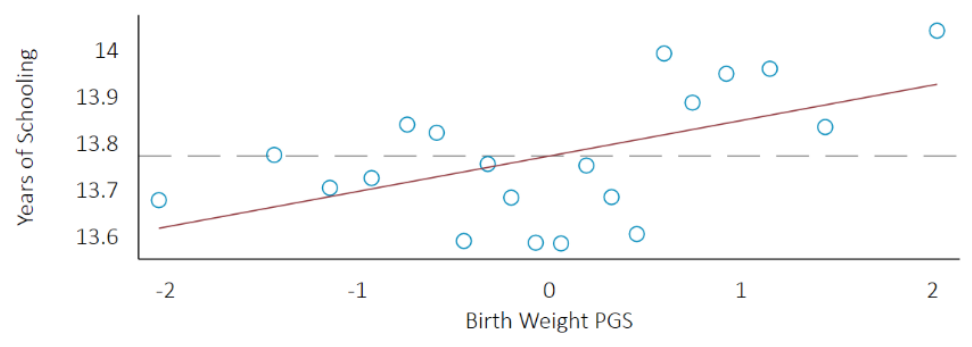

$\mathrm{N}=8350$ individuals
Within Families

B)

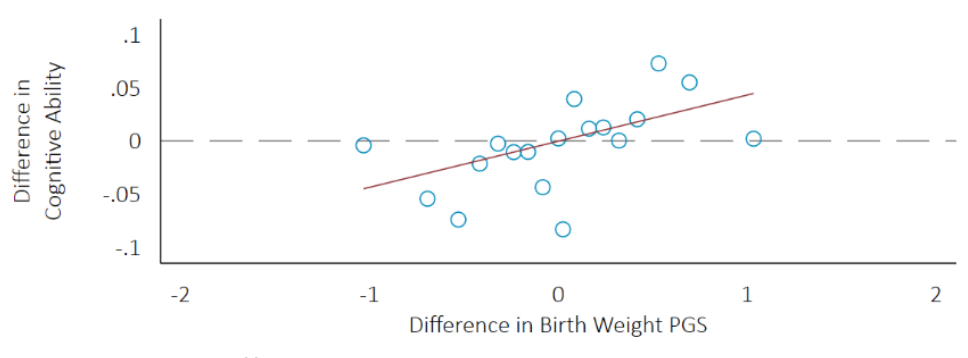

$\mathrm{N}=1731$ sibling pairs

D)

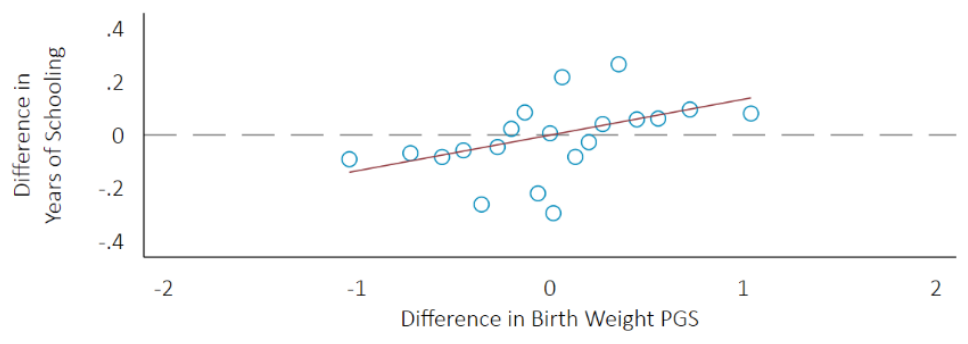

$\mathrm{N}=1980$ sibling pairs

Note. This figure displays data from the Wisconsin Longitudinal Study. Grey dotted line depicts mean value of each plot's outcome variable. Every bin represents roughly 400 individuals (Panels A \& C) or 100 sibling pairs (Panels B \& D). Years of schooling was residualized on a dichotomous indicator for whether an individual is a WLS graduate or randomly selected sibling, birth year, sex, and the first 10 principal components of individual genotype. 
Figure 3

\section{The Association Between Polygenic Score and Predicted Birth Weight Varies by Prenatal Factors}

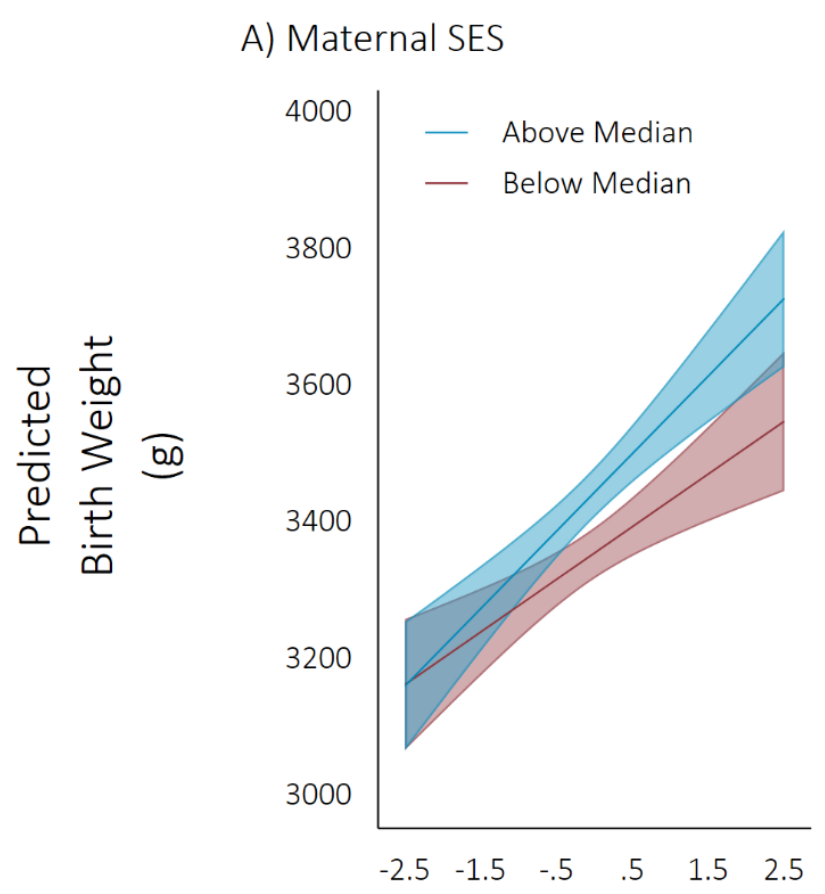

$\mathrm{N}=2007$ individuals
B) Maternal BMI

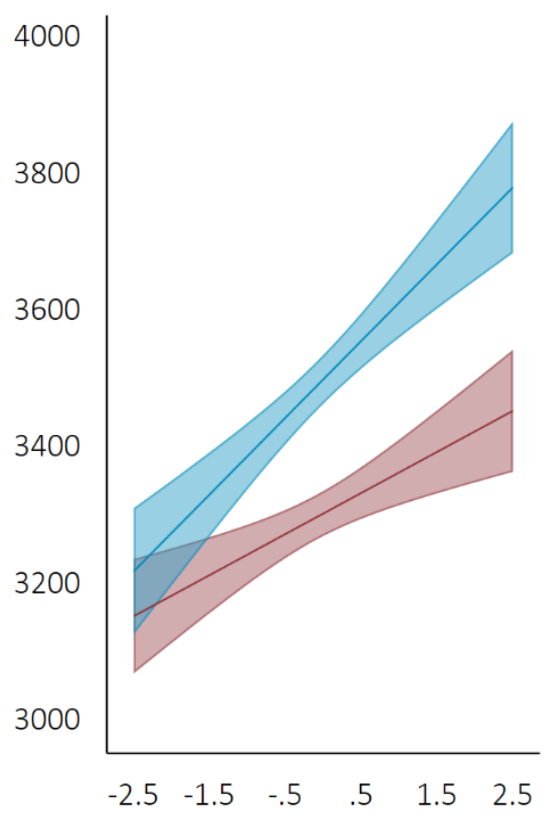

Birth Weight PGS
C) Gestational Length

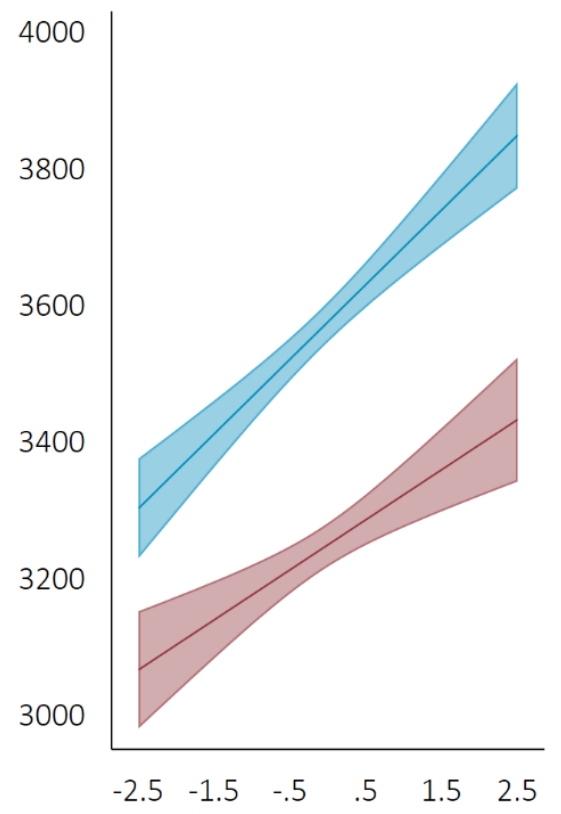

Note. This figure displays data from the Born in Bradford cohort. In addition to the polygenic scores, environmental modifier, and interaction term, the underlying regression model includes controls for sex and the first 10 principal components of individual genotype. See Table A2 of the appendix for the full regression results. Covariates are centered at their mean. Dark lines represent the fitted values and lighter shaded regions represent the $95 \%$ confidence interval. 


\section{Supplementary Tables}

Table A1. Natural Log of Birth Weight Analyses.

A. Birth Weight Regressions

Born in Bradford Wisconsin Longitudinal Study

Birth Weight Polygenic Score

(1)

$0.0266^{* *}$

(2)

$(0.00377)$

$0.0301^{* *}$

(3)

(0.00295)

$0.0243^{* *}$

\section{Family Fixed Effects}

Observations

2007

1930

4934

4190

(0.00850)

Families

Table 4. GxE Regressions.

Born in Bradford

Birth Weight Polygenic Score

0.02

Family Socioeconomic Status

Birth Weight Polygenic Score X Family Socioeconomic Status

(2)

$(0.00416)$

(0.00380)

$\mathrm{X}$

1488

$0.0168 * *$

$(0.00409)$

$0.00742+$

$(0.00402)$

Maternal Body Mass Index

Birth Weight Polygenic Score X Maternal Body Mass Index

Gestational Length

$0.0552 * *$

(0.00238)

0.00342

Birth Weight Polygenic Score X Gestational Length

(0.00223)

\begin{tabular}{lllll} 
Observations & & 1628 & 1740 & 2007 \\
Families & & 1571 & 1685 & 1930 \\
\hline$+p<.10 \quad * p<.05 \quad * * p<.01$ & &
\end{tabular}


Note. This table uses data from the Born and Bradford cohort and the Wisconsin Longitudinal Study. The outcome variable of all regressions is the natural log of birth weight measured in grams. Regressions in the Born the Bradford cohort control for sex and the first 10 principal components of individual genotype. Regressions in the Wisconsin Longitudinal Study control for a dichotomous indicator for whether an individual is a WLS graduate or randomly selected sibling, birth year, sex, and the first 10 principal components of individual genotype. In $\mathrm{BiB}$, birth weight and gestational length were clinically assessed at birth, while maternal body mass index and socioeconomic status were measured at $\sim 27$ weeks gestation. In the Wisconsin Longitudinal Study, birth weight was self-reported retrospectively. Robust standard errors are clustered at the family-level. 
Table A2. Dichotomous GxE Regressions.

Born in Bradford

Birth Weight Polygenic Score

(2)

(2)

$60.12^{* *}$

(3)

High Family Socioeconomic Status

(18.71)

(16.33)

$73.28 * *$

$88.90 * *$

(16.79)

High Family Socioeconomic Status X Birth Weight Polygenic Score

(26.69)

36.38

(26.22)

High Maternal Body Mass Index

$196.5 * *$

High Maternal Body Mass Index X Birth Weight Polygenic Score

High Gestational Length

$326.3^{* *}$

High Gestational Length X Birth Weight Polygenic Score

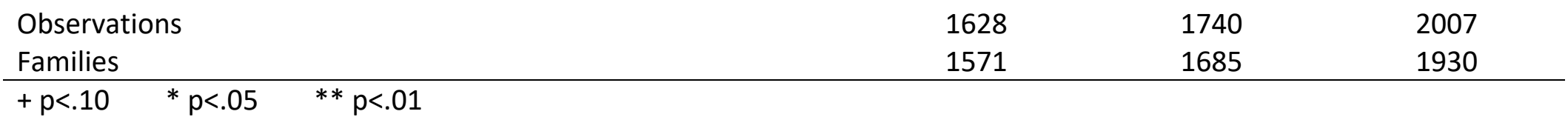

Note. The regressions in this table use data from the Bradford in Bradford cohort and control for sex and the first 10 principal components of individual genotype. Covariates are centered at their mean. Birth weight and gestational length were clinically assessed at birth. Maternal body mass index was clinically assessed at $\sim 27$ weeks gestation. Maternal socioeconomic status is the first principal component of five maternal variables: educational attainment, receipt of mean-tested governmental benefits, neighborhood Index of Multiple Deprivation, partner cohabitation, and home ownership. Gestational length, maternal body mass index, and maternal socioeconomic status are coarsened at their median value into "high" and "low" categories. Robust standard errors are clustered at the family-level. 
Table A3. GxE Development Regressions.

Born in Bradford

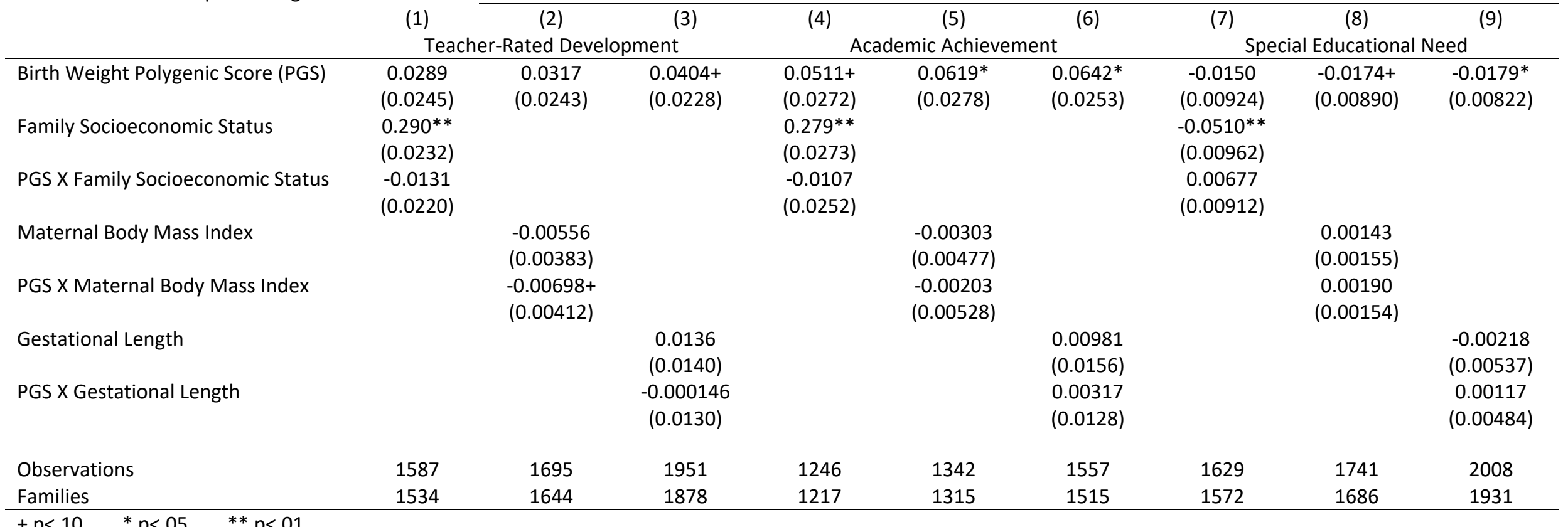

Note. The regressions in this table use data from the Bradford in Bradford cohort and control for sex and the first 10 principal components of individual genotype. Covariates are centered at their mean. Teacher-rated development (age 6), academic achievement (age 8), and special educational need were collected from a student's primary school education record. Gestational length was clinically assessed at birth. Maternal body mass index was clinically assessed at $\sim 27$ weeks gestation. Maternal SES is the first principal component of five maternal variables: educational attainment, receipt of mean-tested governmental benefits, neighborhood Index of Multiple Deprivation, partner cohabitation, and home ownership. Robust standard errors are clustered at the family-level. 